\title{
The Design of Deploying RIS for Millimetre-Wave Signal in Outdoor Environment
}

\author{
Wenming Shi $\mathbb{D}$, Yang Liu, Zhangyou Peng, Mengnan Xu, and Zhonghao Zhang
}

The School of Communication and Information Engineering, Shanghai University, Shanghai 200072, China

Correspondence should be addressed to Wenming Shi; shiwenming@shu.edu.cn

Received 1 December 2020; Revised 2 March 2021; Accepted 8 March 2021; Published 31 March 2021

Academic Editor: Manuel García Sánchez

Copyright (C) 2021 Wenming Shi et al. This is an open access article distributed under the Creative Commons Attribution License, which permits unrestricted use, distribution, and reproduction in any medium, provided the original work is properly cited.

\begin{abstract}
The power coverage of wireless communication is crucial for the quality of communication and cell network evaluation. In this letter, by taking the layout of Reconfigurable Intelligent Surface (RIS) into count, the power coverage area is proposed for a typical outdoor scenario, which has many rectangular buildings. In this approach, via designing the deploying angle, we introduce finding the optimal horizontal and elevation angles of RIS; we adjust RIS so as to get more power coverage. Since the computational complexity of the optimal solution is very high, a low-complexity method is proposed for solving the optimization problem. More specifically, the coverage is showed in the urban environment with RIS assisting. Presenting the numerical results, we can find the power coverage notably improved. It clearly indicates that our experiment is useful to solve the coverage problem for wireless communication.
\end{abstract}

\section{Introduction}

The communication efficiency of wireless networks has greatly improved in the last decade thanks to technological advancement, including millimetre-wave (mmWave) communications. However, the technology faces the practical limitation of shadow effect. The receiving power of receivers behind buildings will reduce $12 \mathrm{~dB}$ than receivers with no obstructions at $28 \mathrm{GHz}$ [1]. The Reconfigurable Intelligent Surface (RIS) is proposed as a promising new solution to address the challenging problem [2-4].

RIS is a planar array composed of a large number of cheap, nearly passive, reflecting elements with parameters. The work [5] maximizes the bit-per-Joule energy efficiency in the downlinks by seeking the RIS phase matrix and the optimal power allocation at the base station (BS). [6] tackles downlink transmitting power minimization for a RIS-aided multiple access network by optimizing both the transmit beamformers at BS and the phase shift matrix at the RIS. To work out the maximization problems of the spectral efficiency in RIS-aided communication systems, [7] presents maximizing the spectral efficiency by optimizing the beamformer at the access point and the RIS phase shifts.
In recent years, the power coverage of mmWave communications attracts more and more attention. Many solutions have been proposed to enhance the coverage area of the RIS-aided communication systems. We present a brief literature review below.

(i) A Subset of the Blockages [8]. The basic idea of this solution is drawing multiple useful system levels. It can enhance objects that act as blockages for the communication links, such as buildings or trees, the coverage probability of the cellular network through providing extra indirect line-of-sight (LoS) links.

(ii) An Achievable Rate Region [9]. By means of investigating the capacity region of a multiple access channel (MAC) with two users sending independent messages to an access point (AP), aided by RIS, it derives a capacity region outer bound for the centralized deployment and the capacity region in closed form for the distributed deployment.

(iii) Reflection Probability [10]. Given the environmental objects modelled with the modified random line 


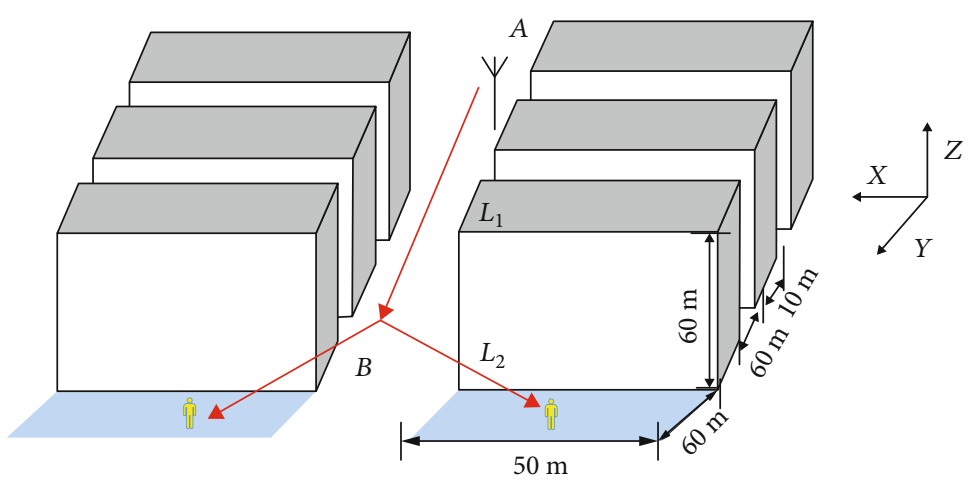

FIGURE 1: Transmission model in the urban scenario based on the mmWave communication.

process of fixed length and random orientations and locations, they propose a new analytical framework that offers one with the probability of a reconfigurable metasurface covering, which is regarded as a reflector for a given transmitter and receiver.

In this letter, we consider the effect of RIS laying angles on the power coverage area in urban communication. Compared to the schemes mentioned above, we propose the design of RIS as well as power coverage that reflects the effect of RIS deploying. We quantify the area which receives energy to get the optimal power coverage area. Specifically, a simple approach is mentioned to solve the problem of finding the optimal angle. Moreover, the contribution of this letter is to replace the RIS with a direction antenna in the urban environmental simulation. This implies that the proposed method is actively yielding a promising solution for the RIS system from deploying.

\section{System Model}

In this section, we present a RIS-aided single-input singleoutput (SISO) communication model. We also describe the targeted receiving power problem formulation.

2.1. Path Loss Model. The results and discussion may be presented separately, or in one combined section, and may optionally be divided into headed subsections. As shown in Figure 1, we consider the transmission model in the urban scenario [11]. The receiving power area of finding the RIS' deploying angle is divided into two areas, and we set the height of receivers as $2 \mathrm{~m}$, where the range of the receivers is from $-55 \mathrm{~m}$ to $-5 \mathrm{~m}$ and $5 \mathrm{~m}$ to $55 \mathrm{~m}$ on the $X$-axis. The width of the receiving power area is $60 \mathrm{~m}$. In this letter, we define the coverage radius of a BS as the geographic area within $350 \mathrm{~m}$. The RIS is envisioned to be installed on the top of a high streetlamp at point $A$ in the figure and in the direct LoS of the BS at point $B$. Therefore, we assume the RIS of the model on the high point where the position of RIS is (LI $\mathrm{S}_{x}, \mathrm{LIS}_{y}, \mathrm{LIS}_{z}$ ). BS is level with RIS, and the position of BS is $\left(\mathrm{Tx}_{x}, \mathrm{Tx}_{y}, \mathrm{Tx}_{z}\right)$. In the same way, the $i$ position of the receivers is regarded as $\left(\mathrm{Rx}_{x}, \mathrm{Rx}_{y}, \mathrm{Rx}_{z}\right)$, and all of the posi- tions form a set $\mathbb{C}$. The receiving power $P_{\mathrm{Rx}}(\mathrm{dBm})$ can be expressed as

$$
\begin{gathered}
P_{\mathrm{Rx}}=P_{\mathrm{Tx}}+G_{\mathrm{Tx}}-P L\left(L_{1}\right)+G-P L\left(L_{2}\right)+G_{\mathrm{Rx}}, \\
P L(L)=-10 \lg \left(\frac{\lambda^{2}}{(4 \pi)^{2} L}\right),
\end{gathered}
$$

where $P L\left(L_{1}\right)$ and $P L\left(L_{2}\right)$ are, respectively, the path loss from the transmitter to RIS and RIS to the receiver. $P_{\mathrm{Tx}}$ denotes the power of the receiver. $G_{\mathrm{Tx}}, G$, and $G_{\mathrm{Rx}}$, respectively, are the gains of transmitting antennas, RIS, and receiving antennas. $\lambda$ is the wavelength.

2.2. Receiver Power Estimation. Figure 2 shows the example of propagation. In allusion to the communication system, the propagation is considered in the far field. When the maximum phase difference of the received signal on the antenna array exceeds $\pi / 9$, we regard the transmitter in the far field. As long as the phase angle of the incident channel offsets the reflection channel's, the receivers can get the maximum power [12]. The received power $P_{\mathrm{Rx}}(\mathrm{mW})$ can be written as [13]

$$
P_{\mathrm{Rx}}=\frac{P_{\mathrm{Tx}} G_{t} G_{r} G M^{2} N^{2} d_{x} d_{y} \lambda^{2} F\left(\theta_{t}, \varphi_{t}\right) F\left(\theta_{r}, \varphi_{r}\right) A^{2}}{64 \pi^{3}\left(L_{1}, L_{2}\right)^{2}} P_{\mathrm{Tx}},
$$

where $d_{x}$ and $d_{y}$, respectively, represent the size of each unit element which are within $\lambda / 10$ and $\lambda / 2$. RIS has $M$ rows and $N$ columns unit elements. $A$ is the amplitude attenuation of RIS. $F\left(\theta_{t}, \varphi_{t}\right)$ and $F\left(\theta_{r}, \varphi_{r}\right)$ represent the incident and reflected normalized power radiation patterns of RIS in the spherical coordinate system, respectively. The normalized power radiation pattern is as follows:

$$
F(\theta, \varphi)= \begin{cases}\cos ^{3} \theta & \theta \in\left[0, \frac{\pi}{2}\right], \varphi \in[0,2 \pi], \\ 0 & \theta \in\left(\frac{\pi}{2}, \pi\right], \varphi \in[0,2 \pi],\end{cases}
$$




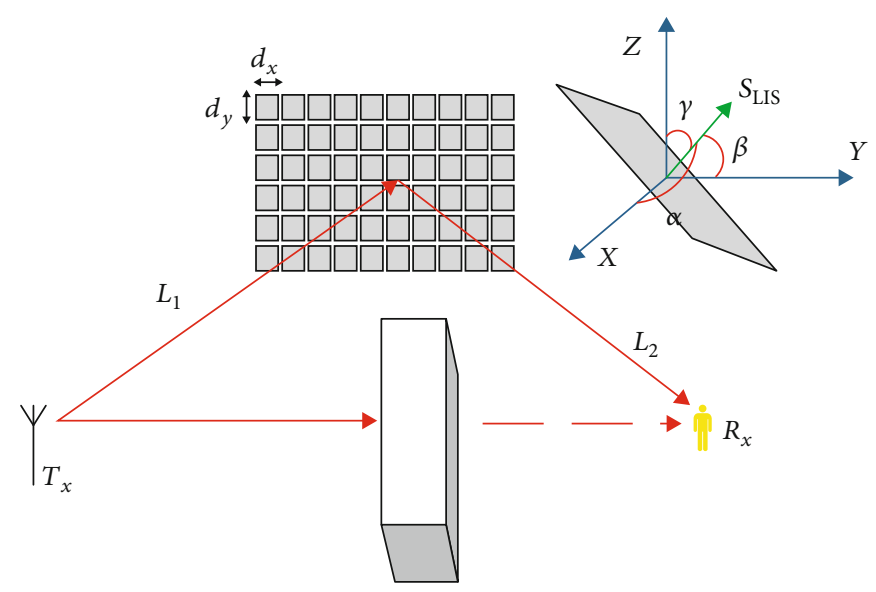

Figure 2: A RIS-aided communication system.
1. Initialize $\Omega_{\min }=-\mathbf{9 0}, \Omega_{\max }=\mathbf{9 0}$
2. Calculate coverage area for $\boldsymbol{\Omega}=\left(\boldsymbol{\Omega}_{\min }+\boldsymbol{\Omega}_{\max }\right) / \mathbf{2}$
3. If coverage area is greater than the result of $\boldsymbol{\Omega}_{\min }$, then set $\boldsymbol{\Omega}_{\min }=\boldsymbol{\Omega}$. If not set $\boldsymbol{\Omega}_{\max }=\boldsymbol{\Omega}$
4. Stop when $\left|\boldsymbol{\Omega}_{\max }-\boldsymbol{\Omega}_{\min }\right|<=\mathbf{1}$.

Algorithm 1

where $\theta$ and $\varphi$, respectively, denote the elevation angle and horizontal angle:

$$
\begin{aligned}
& \cos \theta_{t}=\frac{\mathbf{S}_{\mathrm{LIS}} \cdot \mathbf{S}_{\mathrm{Tx}}}{\left|\mathbf{S}_{\mathrm{LIS}}\right|\left|\mathbf{S}_{\mathrm{Tx}}\right|}, \\
& \cos \theta_{r}=\frac{\mathbf{S}_{\mathrm{LIS}} \cdot \mathbf{S}_{\mathrm{Rx}}}{\left|\mathbf{S}_{\mathrm{LIS}}\right|\left|\mathbf{S}_{\mathrm{Rx}}\right|},
\end{aligned}
$$

where $S_{\text {LIS }}$ denotes the unit normal vector to the RIS plane. $\mathbf{S}_{\mathrm{Tx}}$ and $\mathbf{S}_{\mathrm{Rx}}$ are, respectively, the direction vector of the transmitter to the RIS and the receiver to the RIS.

\section{The Angle of RIS Design}

In this section, acting up to the previous principle, we rotate RIS to acquire the optimized horizontal angle and elevation angle in steps, then layout the RIS in the communication environment.

3.1. The Angle of RIS. As shown in Figure 2, $\alpha, \beta$, and $\gamma$, respectively, are the angle between the $X$-axis, $Y$-axis, $Z$ -axis, and the unit normal vector of the RIS plane. If $\theta \in[0$, $\pi / 2], \mathbf{S}_{\mathrm{Tx}}, \mathbf{S}_{\mathrm{LIS}}$, and $\mathbf{S}_{\mathrm{Rx}}$ are given as

$$
\begin{aligned}
& \mathbf{S}_{\mathrm{Tx}}=\left(\mathrm{Tx}_{x}-\mathrm{LIS}_{x}, \mathrm{Tx}_{y}-\mathrm{LIS}_{y}, \mathrm{Tx}_{z}-\mathrm{LIS}_{z}\right), \\
& \mathbf{S}_{\mathrm{LIS}}=(\cos \alpha, \cos \beta, \cos \gamma), \\
& \mathbf{S}_{\mathrm{Rx}}=\left(\mathrm{Rx}_{x}-\mathrm{LIS}_{x}, \mathrm{Rx}_{y}-\mathrm{LIS}_{y}, \mathrm{Rx}_{z}-\mathrm{LIS}_{z}\right) .
\end{aligned}
$$

Once the positions of the transmitter and RIS are chosen, the next step is to rotate RIS to get the maximum power coverage area. After rotating RIS $\Omega$ around normal direction vector $\mathbf{e}, \mathbf{S}_{\mathrm{Rx}}$ can be expressed as

$$
\mathbf{S}_{\mathrm{LIS}}=\cos (\Omega)\left[\dot{\mathbf{S}}_{\mathrm{LIS}}-\left(\dot{\mathbf{S}}_{\mathrm{LIS}} \cdot \mathbf{e}\right) \mathbf{e}\right]+\sin (\Omega)\left(\dot{\mathbf{S}}_{\mathrm{LIS}} \times \mathbf{e}\right)+\left(\dot{\mathbf{S}}_{\mathrm{LIS}} \cdot \mathbf{e}\right) \mathbf{e}
$$

where $\dot{\mathbf{S}}_{\mathrm{LIS}}$ is the initial normal direction vector of RIS. While we search the horizontal angle, $\mathbf{e}$ is the vertical vector. So long as we find the optimal horizontal angle, the horizontal angle will be fixed and e becomes the horizontal vector in the next step, when we search the elevation angle.

3.2. Low Complexity Approach. As we see in (3) and (4), the coverage area function of $\Omega$, and therefore, it can be maximized by optimizing the rotating angle. The optimal angles of the RISs can be obtained through the following optimization problem:

$$
\begin{gathered}
\underset{\Omega}{\operatorname{maximize}} \sum_{\mathrm{i} \in \mathbb{C}} P_{\mathrm{Rx}}(\Omega) \\
\text { s.t. } P_{\mathrm{Rx}}>P_{\mathrm{Rx}}^{\text {threshold }} \\
-90^{\circ} \leq \Omega \leq 90^{\circ} .
\end{gathered}
$$

We assume the bounds of $\Omega$ as $\Omega_{\min } \leq \Omega \leq \Omega_{\max }$. It can be shown that (11) is a convex problem, and we can solve it efficiently. In Algorithm 1, the bisection method is put forward to solve the problem. In simulation results, the performance of the proposed approach is close to the optimal solution found by exhaustive search. For the first step, the 


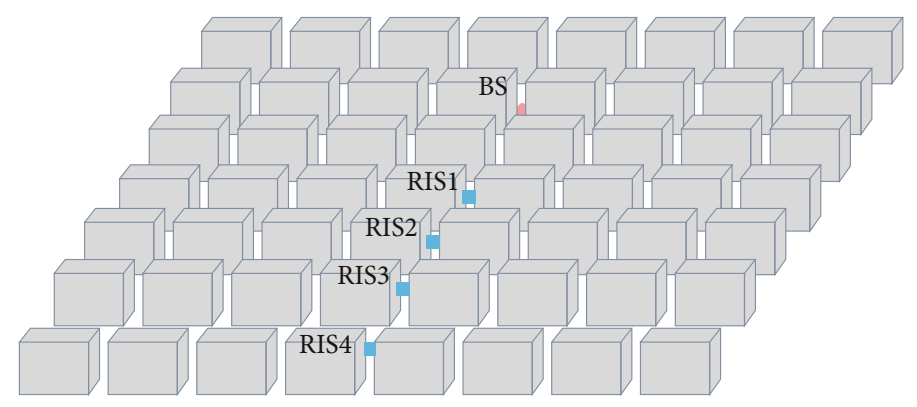

FIgURE 3: The position of transmitter and RIS in the communication environment.

initialized value of $\Omega$ is given to find the optimal in the range. In the second step and third step, the rotating angle is compared and replaced. Only $\left|\Omega_{\max }-\Omega_{\min }\right| \leq 1$, the processing would be stopped. Since we need to find horizontal and elevation angles, the processing should be executed twice to find the angle.

3.3. Parameter Optimization. As shown in Figure 3, the positions of the BS and RISs, respectively, are $(0,0,20)$ and $(0$, $130,20),(0,200,20),(0,270,20)$, and $(0,340,20)$. Due to the complex propagation environment and the better effects of receiving power, we consider the receiving power threshold $P_{\mathrm{Rx}}^{\text {threshold }}$ from -93 to $-99 \mathrm{dBm}$, every two RISs apart $2 \mathrm{dBm}$. Receivers are arranged every one metre. In practice, the material of buildings is concrete.

Furthermore, we observe the power coverage area in the communication environment. The geometry is generated using the Remcom Wireless InSite ray tracing software [2]. In the simulation, we choose the direction antennas [14] to replace the RISs. Note that RISs are deemed to receivers in the BS-RIS links. However, RISs are regarded as transmitters which transmit signals to receivers in the RIS-receivers links. If we ignore the miscellaneous effects that have hardly any influence on the results, $P_{\mathrm{Rx}}$ can be expressed as [15]

$$
P_{\mathrm{Rx}}=M^{2} N^{2} P L_{\mathrm{Rx}}
$$

where $P L_{\mathrm{Rx}}$ denotes the receiving power with no phase when the direction antenna replaces the RIS. When it comes to computing, what counts is cancelling the direction antenna gain. The data rate $C$ in the coverage area is calculated according to the Shannon formula:

$$
C=B \log _{2}\left(1+\frac{P_{\mathrm{Rx}}}{N_{0} B}\right),
$$

where $N_{0}$ denotes the noise power spectral density. $B$ is the bandwidth.

\section{Simulation Results}

Numerical results are provided in this section to demonstrate the effectiveness of the proposed deploying the RIS in the aspect of modulating the angle.

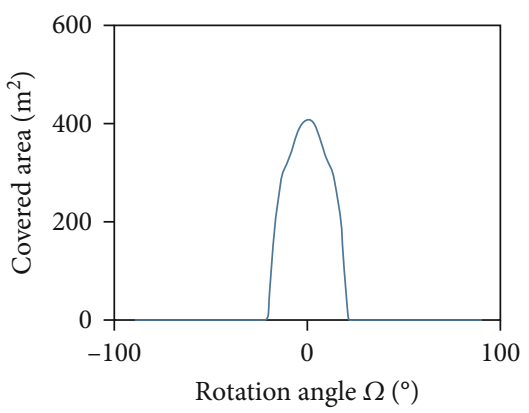

FIGURE 4: RIS $(0,200,20)$ rotate around the horizontal vector. The receiving power is greater than the threshold.

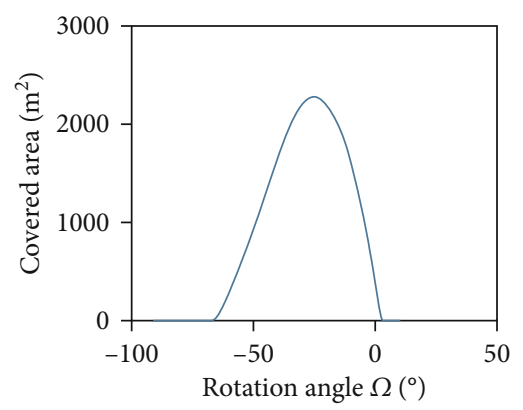

FIgURE 5: Power coverage: the optimal horizontal angle of RIS at $(0,0)$ is $0^{\circ}$. The $X$-axis and $Y$-axis depend on the positions of receivers. Shaded areas are buildings.

4.1. Parameter Selection. There are 56 buildings $(50 \mathrm{~m} \times 10 \mathrm{~m} \times 60 \mathrm{~m})$, and every two adjacent buildings are $60 \mathrm{~m}$ apart. The width of the street is set as $10 \mathrm{~m}$. The carrier frequency is $28 \mathrm{GHz}$, and $P_{\mathrm{Tx}}$ is $20 \mathrm{dBm}$. We choose the halfwave dipole antenna as the antenna of BS and receivers. And the gain is $2.15 \mathrm{dBm}$. $G$ and $A$ are zero. $M$ and $N$ are $100 . d_{x}$ and $d_{y}$ are $\lambda / 2$. Then, replace the $1 \mathrm{~m} \times 1 \mathrm{~m}$ power area with one receiver. $N_{0}$ equals to $-174 \mathrm{dBm} / \mathrm{Hz}$, and $B$ is $40 \mathrm{MHz}$.

As shown in Figure 4, we rotate the RIS $(0,200,20)$ and find that the optimal angle is $0^{\circ}$. Figure 5 shows the power coverage of RIS $(0,200,20)$. The various colour is connected with receiving power. For all of the RISs, the coverage comes to maximum when the horizontal angle is $0^{\circ}$. In other words, the power coverage area becomes maximum as long as BS and RIS are face to face. After getting the optimal horizontal angle, the horizontal angle would be fixed so as to find the 


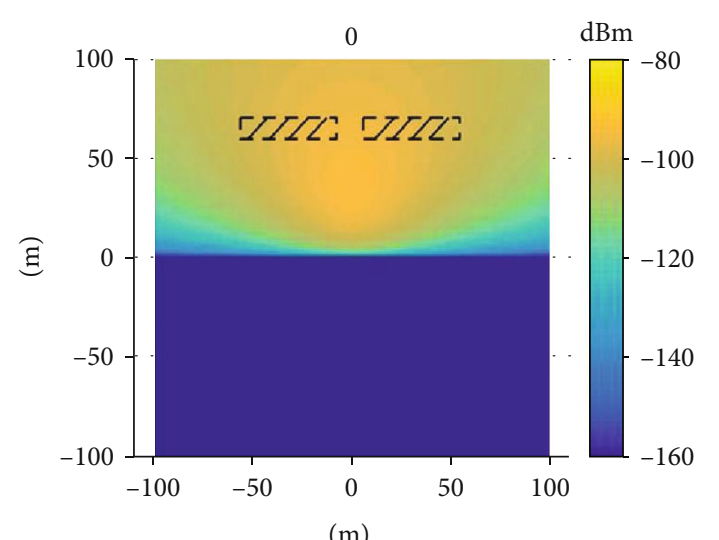

$(\mathrm{m})$

Figure 6: Horizontal angle is set as $0^{\circ}$. RIS $(0,200,20)$ rotate around the horizontal vector. The receiving power is greater than the threshold.

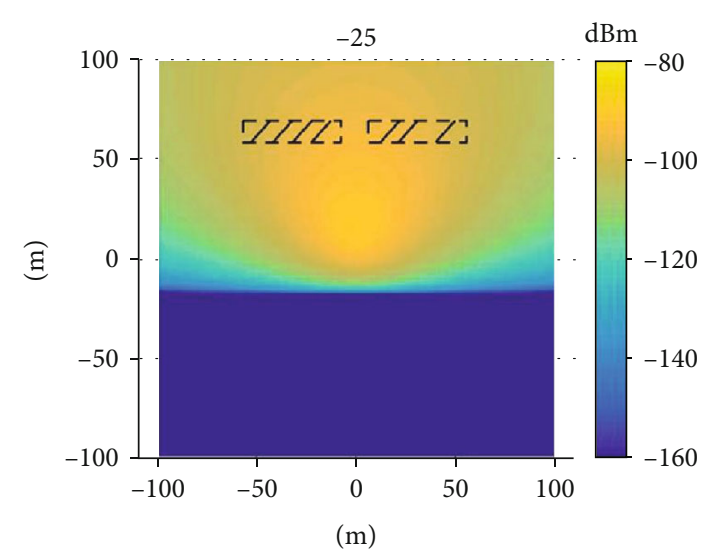

Figure 7: Power coverage: the optimal elevation angle of RIS at $(0,0)$ is $-25^{\circ}$.

optimal elevation angle. Via rotating RIS $(0,200,20)$ right along, we get the power coverage area varying with the rotation angle in Figure 6. Once again, the power coverage is chalked up in Figure 7. According to the abovementioned principle, the optimal horizontal angle of the RIS $(0,130$, $20)$ is $-27^{\circ}$ and the rest of RISs are $-25^{\circ}$. By the proposed algorithm calculation, the angle is approached to the rotating angle. The error is within one degree.

In particular, we consider $\dot{\mathbf{S}}_{\text {LIS }}$ as $(0,-1,0)$. Simultaneously, it is given as the direction vector of no adjusting RIS. It is worth pointing out that we take the receivers for blind spots, whose power is less than $-100 \mathrm{dBm}$ behind buildings. However, provided the power of the area greater than $-100 \mathrm{dBm}$, we regard the area coated [16].

4.2. Result Analysis. Figure 8 shows the power coverage area of BS. Our proposed alternative approach allows us to prove that it is feasible to use one direction antenna instead of all direction antennas on account of ignoring the short distance influence, only if we regard one antenna as the whole antennas when we calculate. In Figure 9, it is depicted the power coverage in the communication system where 4 RISs are

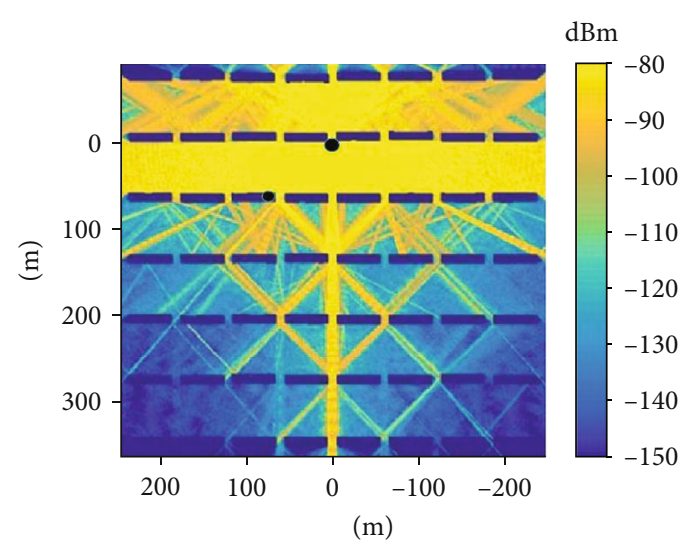

Figure 8: The power coverage of BS. The black point represents BS. The $X$-axis and $Y$-axis depend on the positions.

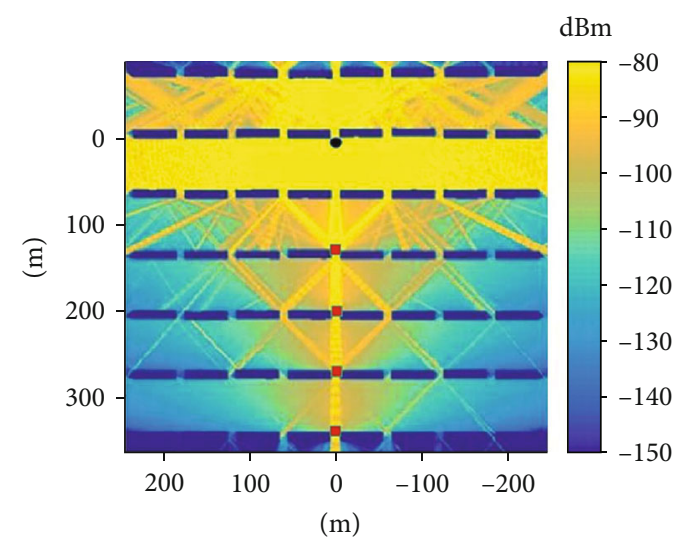

FIgURE 9: The power coverage of joining 4 RISs. The red points represent RISs.

TABLE 1: The performance of different scenarios.

\begin{tabular}{lccc}
\hline Compared scenario & \multicolumn{3}{c}{$\begin{array}{c}\text { Scenario } \\
\text { No RIS }\end{array}$} \\
\hline Coverage rate & $66.24 \%$ & $86.23 \%$ & $90.53 \%$ \\
Blind spots & 12029 & 4873 & 3328 \\
\hline
\end{tabular}

mounted. Specifically, the coverage rate of the whole area reaches $44.89 \%$ when we join the 4 adjusting RISs. According to comparing Figures 8 and 9, it can be clearly observed that the coverage performance of joining 4 RISs is more effectively improved as compared to no RIS. For performance comparison, it can be considered that the compared area is from $-55 \mathrm{~m}$ to $55 \mathrm{~m}$ on the $X$-axis. We demonstrate the performances of various scenarios in the Table 1 , which proves that the power coverage area can be aggrandized by adjusting the angle of RIS. The coverage rate is that the coated area divides by the compared area which is $32400 \mathrm{~m}^{2}$, and it increases $4.3 \%$ by adjusting RISs than no adjusting RISs (the angles of RISs are initial). Furthermore, the blind spots fell a great deal along with adjusting angles in the compared area. As shown in Figure 10, comparing the data rate of optimal angle 


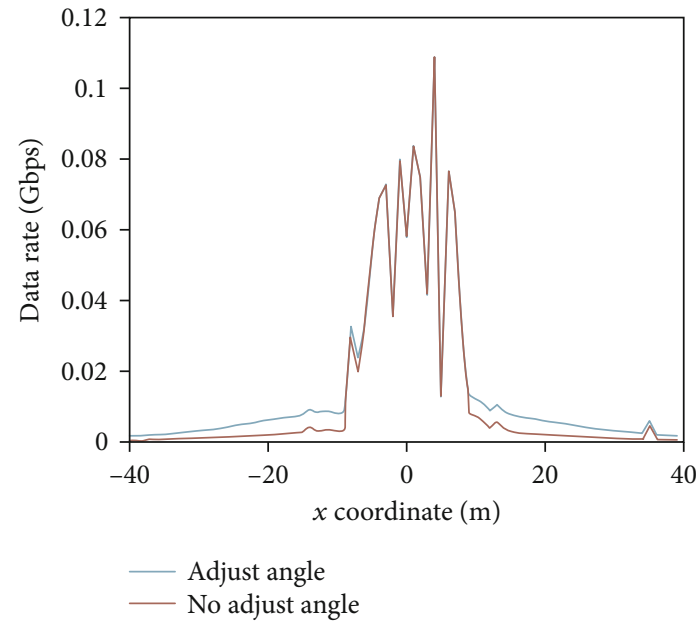

FIgure 10: Data rate performance of a RIS-aided system. $X$ coordinates represent the positions of receivers where the $y$ coordinate is $190 \mathrm{~m}$ and $x$ coordinates are from $-40 \mathrm{~m}$ to $40 \mathrm{~m}$ in Figure 9.

and initial angle, where $y$-coordinate is 190 and $x$-coordinate is from -40 to 40 in Figures 8 and 9, it brings out that RIS, adopting an optimal angle, has remarkable effect.

\section{Conclusions}

In this work, we introduced a layout RIS method, considering the deploying angle of RIS, to improve the power coverage. Then, the power coverage area that evaluates the performance of signal is expressed in many buildings' environment, laid out in an orderly fashion. Furthermore, we analysed the numerical results to confirm that adjusting the deploying angle can achieve more power coverage. Once we know the positions of BS and RISs, we can get the optimal angles. The layout of RIS can be envisioned to be an important energy efficient technology for the future RIS practical applications.

\section{Data Availability}

The data used to support the findings of this study are included within the article.

\section{Conflicts of Interest}

The authors declare that they have no conflicts of interest.

\section{Acknowledgments}

This work is supported in part by the grant from the National Natural Science Foundation of China (under Grant 61701294).

\section{References}

[1] A. S. Kang and R. Vig, "K coverage probability of $5 \mathrm{G}$ wireless cognitive radio network under shadow fading effects," Indonesian Journal of Electrical Engineering and Informatics, vol. 4, no. 3, 2016.
[2] W. Khawaja, O. Ozdemir, Y. Yapici, I. Guvenc, and Y. Kakishima, "Coverage enhancement for mm wave communications using passive reflectors," in 2018 11th Global Symposium on Millimeter Waves (GSMM), pp. 1-6, Boulder, CO, USA, 2018.

[3] Q. U. Nadeem, A. Kammoun, A. Chaaban, M. Debbah, and M. S. Alouini, "Asymptotic analysis of large intelligent surface assisted MIMO communication," 2019, https://arxiv.org/abs/ 1903.08127.

[4] Q. Wu and R. Zhang, "Towards smart and reconfigurable environment: intelligent reflecting surface aided wireless network," IEEE Communications Magazine, vol. 58, no. 1, pp. 106-112, 2019.

[5] C. Huang, A. Zappone, G. C. Alexandropoulos, M. Debbah, and C. Yuen, "Reconfigurable intelligent surfaces for energy efficiency in wireless communication," IEEE Transactions on Wireless Communications, vol. 18, no. 8, pp. 4157-4170, 2019.

[6] M. Fu, Y. Zhou, and Y. Shi, "Intelligent reflecting surface for downlink non-orthogonal multiple access networks," in 2019 IEEE Globecom Workshops (GC Wkshps), pp. 1-6, Waikoloa, HI, USA, 2019.

[7] X. Yu, D. Xu, and R. Schober, "MISO wireless communication systems via intelligent reflecting surfaces," in 2019 IEEE/CIC International Conference on Communications in China (ICCC), pp. 735-740, Changchun, China, 2019.

[8] M. A. Kishk and M. S. Alouini, "Exploiting randomly-located blockages for large-scale deployment of intelligent surfaces," IEEE Journal on Selected Areas in Communications, vol. 39, no. 4, pp. 1043-1056, 2021.

[9] S. Zhang and R. Zhang, "Intelligent reflecting surface aided multiple access: capacity region and deployment strategy," in 2020 IEEE 21st International Workshop on Signal Processing Advances in Wireless Communications (SPAWC), pp. 1-5, Atlanta, GA, USA, 2020.

[10] M. Di Renzo and J. Song, "Reflection probability in wireless networks with metasurface-coated environmental objects: an approach based on random spatial processes," EURASIP Journal on Wireless Communications and Networking, vol. 2019, no. 1, p. 15, 2019.

[11] K. Haneda, J. Zhang, L. Tan et al., "5G 3GPP-like channel models for outdoor urban microcellular and macrocellular environments," in 2016 IEEE 83rd Vehicular Technology Conference (VTC Spring), pp. 1-7, Nanjing, China, 2016.

[12] A. Taha, M. Alrabeiah, and A. Alkhateeb, "Enabling large intelligent surfaces with compressive sensing and deep learning," 2019, https://arxiv.org/abs/1904.10136.

[13] W. Tang, M. Z. Chen, X. Chen et al., "Wireless communications with reconfigurable intelligent surface: path loss modeling and experimental measurement," IEEE Transactions on Wireless Communications, vol. 20, 2020.

[14] L. L. Rainwater, "Steerable Directional Antenna," U.S. Patent No. 4,345,256. 17 Aug. 1982.

[15] P. Chai and L. Zhang, "Indoor radio propagation models and wireless network planning," in 2012 IEEE International Conference on Computer Science and Automation Engineering (CSAE), pp. 738-741, Zhangjiajie, China, 2012.

[16] E. Basar, "Reconfigurable intelligent surface-based index modulation: a new beyond MIMO paradigm for 6G," IEEE Transactions on Communications, vol. 68, no. 5, pp. 3187-3196, 2020. 